\title{
Investigation of the 1+1 dimensional Thirring model using the method of matrix product states
}

\author{
Mari Carmen Bañuls \\ Max Planck Institut für Quantenoptik, Garching 86748, Germany \\ E-mail: banulsmempq.mpg.de
}

\section{Krzysztof Cichy}

Faculty of Physics, Adam Mickiewicz University, ul. Umultowska 85, 61-614 Poznań, Poland

E-mail: krzysztof.cichyegmail.com

\section{Ying-Jer Kao}

Department of Physics, National Taiwan University, Taipei 10617 Taiwan

E-mail: yjkao@phys.ntu.edu.tw

\section{C.-J. David Lin*}

Institute of Physics, National Chiao-Tung University, Hsinchu 30010, Taiwan

Centre for High Energy Physics, Chung-Yuan Christian University, Chung-Li, 32032, Taiwan

E-mail: dlin@mail.nctu.edu.tw

\section{Yu-Ping Lin}

Department of Physics, University of Colorado, Boulder, CO 80309, USA

E-mail: Yuping.Linecolorado.edu

\section{David T.-L Tan}

Institute of Physics, National Chiao-Tung University, Hsinchu 30010, Taiwan

E-mail: tanlin2013.py04g@nctu.edu.tw

\begin{abstract}
We present preliminary results of a study on the non-thermal phase structure of the $(1+1)$ dimensional massive Thirring model, employing the method of matrix product states. Through investigating the entanglement entropy, the fermion correlators and the chiral condensate, it is found that this approach enables us to observe numerical evidence of a Kosterlitz-Thouless phase transition in the model.
\end{abstract}

The 36th Annual International Symposium on Lattice Field Theory - LATTICE2018

22-28 July, 2018

Michigan State University, East Lansing, Michigan, USA.

\footnotetext{
* Speaker.
} 


\section{Introduction}

For the past few years, tensor-network (TN) methods for quantum many-body systems have been attracting attention from practitioners of lattice field theory. These methods feature several appealing qualities, such as the opportunity for studying real-time dynamics of quantum field theories (QFT's), and the possibility of solving the sign problem. In addition to exploring the formulation of various QFT's as TN's, there have also been efforts on applying existing techniques to lattice field theory computations. See. e.g., Refs. [1,2] for a summary of recent activities.

In this article, we present progress of our investigation for the non-thermal phase structure of (1+1)-dimensional massive Thirring model using the formulation of matrix product states (MPS). The action of this model is

$$
S_{\mathrm{Th}}[\psi, \bar{\psi}]=\int d^{2} x\left[\bar{\psi} i \gamma^{\mu} \partial_{\mu} \psi-m \bar{\psi} \psi-\frac{g}{2}\left(\bar{\psi} \gamma_{\mu} \psi\right)\left(\bar{\psi} \gamma^{\mu} \psi\right)\right],
$$

where $m$ and $g$ denote the fermion mass and the dimensionless four-fermion coupling constant. As shown by Coleman [3] and Mandelstam [4], the model in Eq. (1.1) is S-dual to the sine-Gordon (SG) theory in the sector of zero total fermion number. The SG theory is also known to be dual to the classical two-dimensional XY model $[5]^{1}$. Therefore, a Kosterlitz-Thouless (KT) phase transition [7] is expected in the Thirring model. Hints for this phase structure are also observed in the Thirring-model renormalisation group (RG) equations up to $\mathscr{O}\left(\mathrm{m}^{3}\right)$ in perturbation theory [8],

$$
\beta_{g} \equiv \mu \frac{d g}{d \mu}=-64 \pi \frac{m^{2}}{\Lambda^{2}}, \beta_{m} \equiv \mu \frac{d m}{d \mu}=\frac{-2\left(g+\frac{\pi}{2}\right)}{g+\pi} m-\frac{256 \pi^{3}}{(g+\pi)^{2} \Lambda^{2}} m^{3},
$$

which bear the same characteristics as the Kosterlitz scaling equations for the XY model [9]. For $g<-\pi / 2$, RG transformation evolves the theory to the massless Thirring model which is a conformal field theory (CFT). In this regime, the dual SG model is shown to be a free bosonic QFT at low energy. At $g>-\pi / 2$, the $\bar{\psi} \psi$ operator in Eq. (1.1) is relevant and the theory contains a scale.

In the current project, we demonstrate that the MPS formulation can be employed to perform numerical lattice calculations for investigating the phase structure of the massive Thirring model. This is the foundation work for our future study of real-time dynamics of the above phase transition. Exploratory results of this project were already presented in Ref. [10].

\section{Massive Thirring model and the $X X Z$ quantum spin chain}

Equation (1.1) describes the classical action of the Thirring model. To obtain the operator formalism for this model as a QFT, it is necessary to incorporate effects of the anomalous breaking of the vector symmetry in two dimensions [11]. In this procedure, the construction of the Hamiltonian can be achieved by studying the operator relations that are satisfied by the energy-momentum tensor [12]. Such relations result in the continuum Thirring-model Hamiltonian operator,

$$
H_{\mathrm{Th}}=\int d x\left[-i Z_{\psi}(g) \bar{\psi} \gamma^{1} \partial_{1} \psi+m_{0} \bar{\psi} \psi+\frac{g}{4}\left(\bar{\psi} \gamma^{0} \psi\right)^{2}-\frac{\tilde{g}(g)}{2}\left(\bar{\psi} \gamma^{1} \psi\right)^{2}\right]
$$

\footnotetext{
${ }^{1}$ In addition to establishing the equivalence between partition functions of the SC theory and the two-dimensional Coulomb gas, it was also shown that the XY-model vortices can be manifested as SG solitons [5, 6].
} 
where $m_{0}$ is the bare mass, $Z_{\psi}(g)$ is the wavefunction renormalisation constant [13, 14], and $\tilde{g}(g)=$ $g(g+\pi) /(2 g+\pi)$.

Since we work in the Hamiltonian formalism, discretisation of the theory only has to be carried out in the spatial direction. This is achieved using staggered fermions $[15,16]$. In this strategy, one of the two components, $\psi_{1}$ and $\psi_{2}$, of the Dirac fermion is removed on each lattice site. This is implemented via $\psi_{1}(x) \rightarrow \frac{1}{\sqrt{a}} c_{2 n}, \psi_{2}(x) \rightarrow \frac{1}{\sqrt{a}} c_{2 n+1}$, where $a$ is the lattice spacing, and $c_{i}$ is a one-component fermionic degree of freedom at the $i$-th site of the one-dimensional spatial lattice. Choosing the standard representation of the Dirac matrices, $\gamma^{0}=\sigma^{z}, \gamma^{1}=i \sigma^{y}, \gamma^{5}=\sigma^{x}\left(\left\{\sigma^{i}\right\}\right.$ are the Pauli matrices), we obtain the staggered Thirring-model Hamiltonian operator on the lattice,

$$
H_{\mathrm{Th}}^{(\mathrm{latt})}=-\frac{i}{2 a} Z_{\psi}(g) \sum_{n=0}^{N-2}\left(c_{n}^{\dagger} c_{n+1}-c_{n+1}^{\dagger} c_{n}\right)+m_{0} \sum_{n=0}^{N-1}(-1)^{n} c_{n}^{\dagger} c_{n}+\frac{\tilde{g}(g)}{2 a} \sum_{n=0}^{N-2} c_{n}^{\dagger} c_{n} c_{n+1}^{\dagger} c_{n+1},
$$

where $N$ is the total number of lattice sites. Since we are only discretising the spatial direction, the fermion doubling problem is completely evaded in staggered fermions. Therefore, $H_{\mathrm{Th}}^{(\text {latt })}$ is describing one "flavour" of fermion at the effective lattice spacing $2 a$.

It is challenging to perform simulations with the fermionic degrees of freedom in Eq. (2.2). To proceed, we employ the Jordan-Wigner (JW) transformation to represent the model using spin matrices. This results in a one-dimensional quantum XXZ spin chain coupled to uniform and staggered external magnetic fields. The system is described by the Hamiltonian [17],

$$
\begin{array}{r}
H_{\mathrm{XXZ}}=\frac{v(g)}{a} \bar{H}_{\mathrm{sim}}, \text { with } \bar{H}_{\mathrm{sim}}=-\frac{1}{2} \sum_{n=0}^{N-2}\left(S_{n}^{+} S_{n+1}^{-}+S_{n+1}^{+} S_{n}^{-}\right)+a \tilde{m}_{0} \sum_{n=0}^{N-1}(-1)^{n}\left(S_{n}^{z}+\frac{1}{2}\right) \\
+\Delta(g) \sum_{n=0}^{N-1}\left(S_{n}^{z}+\frac{1}{2}\right)\left(S_{n+1}^{z}+\frac{1}{2}\right),
\end{array}
$$

where $S_{n}^{ \pm}=S_{n}^{x} \pm i S_{n}^{y}$ is the spin matrix $\left(S_{n}^{i}=\sigma^{i} / 2\right)$ at the $n$-the lattice site, and $\left[S_{n}^{i}, S_{m}^{j}\right]=0$ when $n \neq m$. The functions $v(g)$ and $\Delta(g)$ are the lattice counterparts of $Z_{\psi}(g)$ and $\tilde{g}(g)$ in Eq. (2.1) [17],

$$
v(g)=\left(\frac{\pi-g}{\pi}\right) / \sin \left(\frac{\pi-g}{2}\right), \Delta(g)=\cos \left(\frac{\pi-g}{2}\right),
$$

and $\tilde{m}_{0}=m_{0} / v(g)$ is the rescaled bare mass.

\section{Matrix product states and simulation details}

We continue the phase-structure study with the ground state search in the Hamiltonian obtained by modifying $\bar{H}_{\text {sim }}$ in Eq. (2.3),

$$
\bar{H}_{\text {sim }}^{\text {penalty }}=\bar{H}_{\text {sim }}+\lambda\left(\sum_{n=0}^{N-1} S_{n}^{z}\right)^{2}
$$

where $\lambda$ is a parameter chosen to be 100 in this work. The "penalty term" guarantees that $\sum_{n=0}^{N-1} S_{n}^{z}=$ 0 for the ground state determined with a variational search strategy [18]. Employing the JW transformation, it can be demonstrated that this corresponds to constraining the Thirring model in the sector of zero total fermion number, in which the duality with the SG theory is valid. 
The size of the Hilbert space in a quantum spin chain increases exponentially with the system size, $N$. This makes numerical computations very challenging in practice. The MPS method is an efficient, entanglement-based, way to truncate the Hilbert space, and can be combined with the approach ála density matrix RG (DMRG) $[19,20]$ for obtaining the ground state. Its implementation for representing a quantum state of $N$ spins $\left(\sigma_{1}, \ldots, \sigma_{N}\right)$ in one dimension can be summarised as

$$
|\Psi\rangle=\sum_{\sigma_{1}, \ldots, \sigma_{N}} c_{\sigma_{1} \ldots \sigma_{N}}\left|\sigma_{1} \ldots \sigma_{N}\right\rangle \stackrel{\mathrm{MPS}}{\longrightarrow} \sum_{\sigma_{1}, \ldots, \sigma_{N}} M^{\sigma_{1}} M^{\sigma_{2}} \ldots M^{\sigma_{N-1}} M^{\sigma_{N}}\left|\sigma_{1} \ldots \sigma_{N}\right\rangle,
$$

where each $M^{\sigma_{n}}$ is a matrix with maximally-allowed dimension (the bond dimension) being $D$. The strategy in (3.2) involves an approximation of the rank $-N$ tensor, $c_{\sigma_{1} \ldots \sigma_{N}}$, by $N$ rank-3 tensors, $\left\{M_{i, j}^{\sigma_{n}}\right\}$. This implies a truncation of the Hilbert space which can be achieved via singular value (SV) decomposition with respect to each cut of the chain, in which only the largest $D$ SV's are kept. This programme often allows one to capture low-energy physics with small enough $D$ in practice.

To carry out the DMRG search for the ground state of $\bar{H}_{\text {sim }}^{\text {penalty }}$, we also need the matrix product operator for this Hamiltonian. Through explicit construction, we find $\left[\beta_{n} \equiv \Delta(g)+(-1)^{n} \tilde{m}_{0} a\right]$,

$$
\bar{H}_{\text {sim }}^{\text {penalty }}=\prod_{n=0}^{N-1} W^{[n]},
$$

where

$$
\begin{aligned}
& W^{[0]}=\left(\begin{array}{lllllll}
\mathbb{I} & -\frac{1}{2} S_{0}^{+} & -\frac{1}{2} S_{0}^{-} & 2 \lambda S_{0}^{z} & \Delta(g) S_{0}^{z} & \beta_{0} S_{0}^{z}+\frac{\lambda}{4}
\end{array}\right), \\
& W^{[N-1]}=\left(\begin{array}{lllllll}
\beta_{N-1} S_{N-1}^{z}+\frac{\lambda}{4} & S_{N-1}^{-} & S_{N-1}^{+} & S_{N-1}^{z} & S_{N-1}^{z} & \mathbb{I}
\end{array}\right)^{T},
\end{aligned}
$$

and $\left.W^{[n]}\right|_{2 \leq n \leq N-2} \equiv W_{\text {bulk }}^{[n]}$ is a $6 \times 6$ operator-valued matrix,

$$
\begin{aligned}
& \left(W_{\text {bulk }}^{[n]}\right)_{1 j}=\left(\left.W^{[0]}\right|_{S_{0}^{+,-, z}, \beta_{0} \rightarrow S_{n}^{+,-, z}, \beta_{n}}\right)_{1 j},\left(W_{\text {bulk }}^{[n]}\right)_{i 6}=\left(\left.W^{[N-1]}\right|_{S_{N-1}^{+,-,}, \beta_{N-1} \rightarrow S_{n}^{+,-, z}, \beta_{n}}\right)_{i 1}, \\
& \left(W_{\text {bulk }}^{[n]}\right)_{44}=\mathbb{I} \text {, and all other }\left(W_{\text {bulk }}^{[n]}\right)_{i j}=0 .
\end{aligned}
$$

In this work, we perform simulations at four system sizes, $N=400,600,800,1000$. This enables us to extrapolate our results to the infinite-volume limit. To carry out the initial scanning for studying the phase structure, twenty-one values of the bare four-fermion coupling are chosen, straddling the range $-0.9 \leq \Delta(g) \leq 1.0$, and five values of $a \tilde{m}_{0}(0,0.1,0.2,0.3,0.4)$ are selected. As explained in the next section, simulations at smaller fermion masses are needed, and we have been generating data at $a \tilde{m}_{0}=0.005,0.01,0.02,0.03,0.04,0.06,0.08,0.13,0.16$.

We commence the DMRG ground-state search at $D=50$, with a set of random initial matrices, $\left\{M^{\sigma_{n}}\right\}$. Results at six larger bond dimension, $D=100,200, \ldots, 600$ are then subsequently obtained by using the $\left\{M^{\sigma_{n}}\right\}$ at smaller $D$ as the initial input. Having data at many values of the bond dimension enables us to investigate the infinite $-D$ limit. The variational update of the MPS is performed locally [20] by sweeping over the lattice. The target precision of the ground-state energy is $10^{-8}$ in lattice units. Figure 1 shows examples of how the DMRG search converges at two choices of the bare parameters. Notice that significantly different convergence properties are observed in our simulations, leading to hints that there are distinct phases in the theory. 

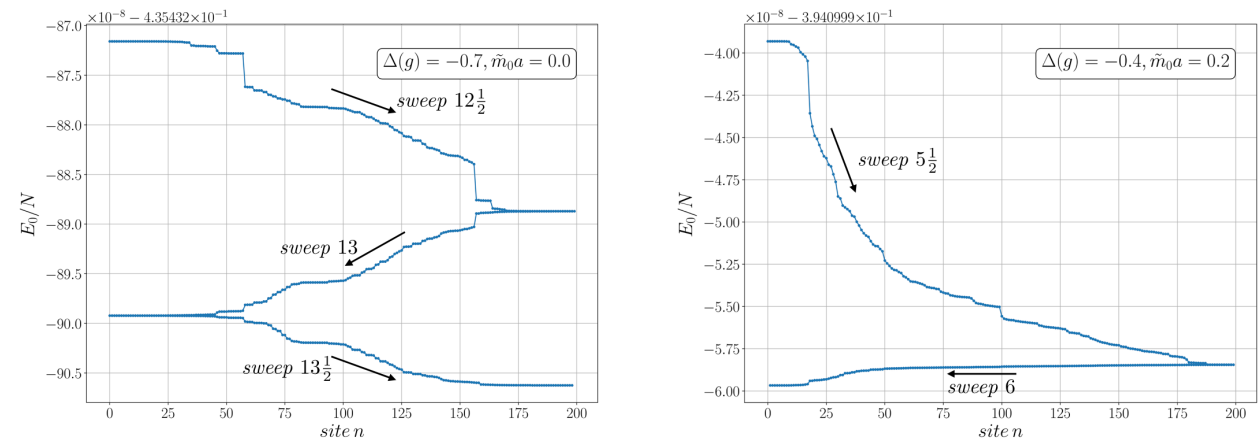

Figure 1: Convergence of DMRG with two choices of $\left[\Delta(g), a \tilde{m}_{0}\right]$ at $N=200$ and $D=50$.



(a)

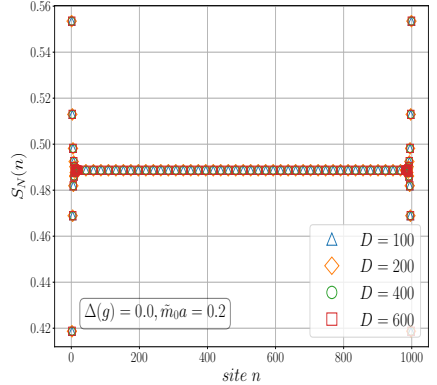

(b)



(c)

Figure 2: $S_{N}(n)$ at $N=1000$ plotted against (a) and (b): $n$ and (c): $\frac{1}{6} \ln \left[\frac{N}{\pi} \sin \left(\frac{\pi n}{N}\right)\right]$.

\section{Numerical results for the phase structure of the massive Thirring model}

To investigate the non-thermal phase structure of the massive Thirring model, we first examine the von Neumann entanglement entropy, computed by dividing the system of size $N$ into two parts between sites $n$ and $n+1$. Denoting this entropy by $S_{N}(n)$, a scaling law is valid when the theory is conformal [21],

$$
S_{N}(n)=\frac{c}{6} \ln \left[\frac{N}{\pi} \sin \left(\frac{\pi n}{N}\right)\right]+k \quad(n=0,1,2, \ldots, N-1),
$$

where $c$ is the central charge, and $k$ is a constant. Figure 2(a) demonstrates that this scaling is manifested at $a \tilde{m}_{0}=0.2$ and $\Delta(g)=-0.88$, with large enough $D$, and Fig. 2(c) shows that $c=1$ in this case, indicating that the theory in this regime is equivalent to a free bosonic QFT. For the same choice of $a \tilde{m}_{0}=0.2$, the behaviour in Eq. (4.1) is not realised when $\Delta(g)$ is increased beyond a critical value. This is evidenced by the plot in Fig. 2(b). Such a feature persists in all our results at $a \tilde{m}_{0} \neq 0$, while we find that the theory is always conformal with $c=1$ when $a \tilde{m}_{0}=0$.

Next, we study the correlator of left-handed fermion fields, $\psi_{\mathrm{L}}(0)$ and $\psi_{\mathrm{L}}^{\dagger}(\hat{r})$, with $\hat{r} \equiv r / a$,

$$
\bar{G}(\hat{r}) \equiv G(\hat{r}) / G(0), \text { where } G(\hat{r})=\left\langle\psi_{\mathrm{L}}^{\dagger}(\hat{r}) \psi_{\mathrm{L}}(0)\right\rangle \stackrel{\text { JW transformation }}{\longrightarrow}\left\langle S_{\hat{r}}^{+} \mathrm{e}^{i \pi \sum_{j=1}^{\hat{\gamma}-1} S_{j}^{z}} S_{0}^{-}\right\rangle .
$$

This correlator is bosonised to the soliton correlator in the SG model [4], and exhibits power law in $\hat{r}$ in the conformal phase, while decays exponentially when the theory is gapped [22]. From Fig. 3, 



Figure 3: Fermion (soliton) correlators at $a \tilde{m}_{0}=0.0$ and 0.2 .

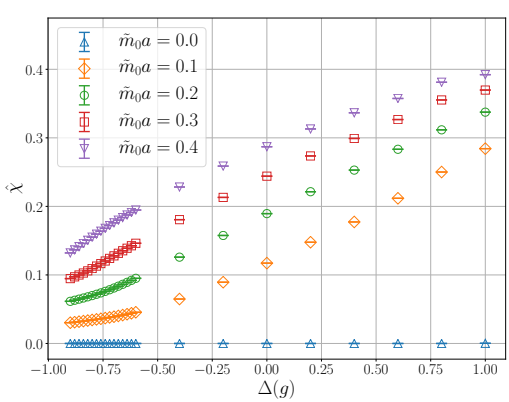

(a)

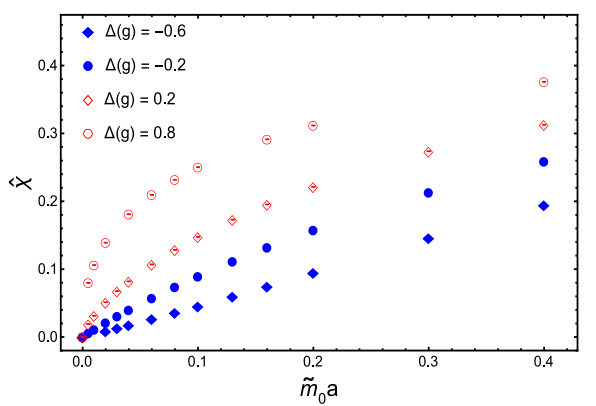

(b)

Figure 4: Results of chiral condensate in the limit of infinite bond dimension and system size.

we see evidence that the model is conformal when $a \tilde{m}_{0}=0$. For $a \tilde{m}_{0}=0.2$ (also observed for other values of non-zero $a \tilde{m}_{0}$ ), there is a phase transition. Furthermore, the exponent for the power-law decay of this correlator depends on $g$. This leads to hints that the transition is of KT-type.

The last quantity we investigate is the chiral condensate, $\hat{\chi}$. Under the JW transformation,

$$
\hat{\chi} \equiv \frac{a}{N} \sum_{n=0}^{N-1}\langle\bar{\psi}(n) \psi(n)\rangle \stackrel{\mathrm{JW} \text { transformation }}{\longrightarrow} \frac{1}{N}\left\langle\sum_{n=0}^{N-1}(-1)^{n} S_{n}^{z}\right\rangle .
$$

Results for this quantity at $a \tilde{m}_{0}=0,0.1,0.2,0.3$ and 0.4 , extrapolated to infinite $D$ and $N$, are displayed in Fig. 4(a). We also performed direct infinite-size simulations for $a \tilde{m}_{0}=0$ using the uniform MPS method [23], and obtain $\hat{\chi}=0$. To confirm that we are observing a KT transition, it is crucial to verify that the chiral condensate computed with non-vanishing mass extrapolates to zero in the massless limit [22]. Figure 4(b) shows this behaviour in both gapped and conformal phases $^{2}$. It also demonstrates the importance of having results at $a \tilde{m}_{0}<0.1$. Currently we are performing more simulations in this regime.

\section{Conclusion and outlook}

In this article, we present our investigation of the non-thermal phase structure of the $(1+1)$ dimensional massive Thirring model, employing the MPS strategy. We find that this approach can

\footnotetext{
${ }^{2} \mathrm{~A}$ vanishing condensate at $a \tilde{m}_{0}=0$ is consistent with the Mermin-Wagner-Coleman theorem [24, 25].
} 
be successfully applied to such studies, and numerical evidence for an expected KT phase transition is observed. To further confirm this observation, we are currently examining the spectrum of the transfer matrix in this model. Since the MPS method is formulated in the Hamiltonian formalism, in the near future we will also use this technique to probe real-time dynamics of this phase transition.

\section{Acknowledgments}

MCB is supported by the QUANTERA project QTFLAG. KC was partially supported by the DFG project nr. CI 236/1-1. YJK, CJDL, YPL and DTLT acknowledge funding from Taiwanese MoST via grants 105-2628-M-009-003-MY4, 105-2112-M-002 -023 -MY3, 104-2112-M-002 $022-\mathrm{MY} 3$.

\section{References}

[1] M. C. Bañuls, K. Cichy, J. I. Cirac, K. Jansen and S. Kühn, PoS(LATTICE2018) 022.

[2] M. C. Bañuls et al., EPJ Web Conf. 137 (2017) 04001 [arXiv:1611.04791 [hep-lat]].

[3] S. R. Coleman, Phys. Rev. D 11 (1975) 2088.

[4] S. Mandelstam, Phys. Rev. D 11 (1975) 3026.

[5] J. Jose, Phys. Rev. D 14 (1976) 2826.

[6] K. Huang and J. Polonyi, Int. J. Mod. Phys. A 6 (1991) 409.

[7] J. M. Kosterlitz and D. J. Thouless, J. Phys. C 6 (1973) 1181.

[8] J. Zinn-Justin, hep-ph/0005272.

[9] J. M. Kosterlitz, J. Phys. C 7 (1974) 1046.

[10] M. C. Bañuls et al., EPJ Web Conf. 175 (2018) 11017 [arXiv:1710.09993 [hep-lat]].

[11] J. S. Schwinger, Phys. Rev. 128 (1962) 2425.

[12] C. R. Hagen, Nuovo Cimento B51 (1967) 169.

[13] A. H. Mueller and T. L. Trueman, Phys. Rev. D 4 (1971) 1635.

[14] M. Gomes and J. H. Lowenstein, Nucl. Phys. B 45 (1972) 252.

[15] T. Banks, L. Susskind and J. B. Kogut, Phys. Rev. D 13 (1976) 1043.

[16] L. Susskind, Phys. Rev. D 16 (1977) 3031.

[17] A. Luther, Phys. Rev. B 14 (1976) 2153.

[18] M. C. Bañuls, K. Cichy, K. Jansen and J. I. Cirac, JHEP 1311 (2013) 158 [arXiv:1305.3765 [hep-lat]].

[19] S. R. White, Phys. Rev. Lett. 69 (1992) 2863.

[20] U. Schollwock, Ann. Phys. 326 (2011) 96.

[21] P. Calabrese and J. L. Cardy, J. Stat. Mech. 0406 (2004) P06002 [hep-th/0405152].

[22] E. Witten, Nucl. Phys. B 145 (1978) 110.

[23] V. Zauner-Stauber et al., Phys. Rev. B 97 (2018) no.4, 045145 [arXiv:1701.07035 [quant-ph]].

[24] N. D. Mermin and H. Wagner, Phys. Rev. Lett. 17 (1966) 1133.

[25] S. R. Coleman, Commun. Math. Phys. 31 (1973) 259. 